\title{
La 'negra nieves' ou le racisme à fleur de peau. Regards croisés sur une caricature
}

La 'negra nieves' o el racismo a flor de piel. Miradas cruzadas sobre una caricatura

'Black nieves' or racism based on appearances. Crossed glances on a caricature

\section{Elisabeth Cunin}

\section{OpenEdition}

Journals

Édition électronique

URL : http://journals.openedition.org/bifea/6164

DOI : $10.4000 /$ bifea.6164

ISSN : 2076-5827

\section{Éditeur}

Institut Français d'Études Andines

\section{Édition imprimée}

Date de publication : 1 septembre 2003

Pagination : 237-262

ISSN : 0303-7495

\section{Référence électronique}

Elisabeth Cunin, "La 'negra nieves' ou le racisme à fleur de peau. Regards croisés sur une caricature», Bulletin de l'Institut français d'études andines [En ligne], 32 (2) | 2003, mis en ligne le 08 mai 2003, consulté le 01 décembre 2020. URL : http://journals.openedition.org/bifea/6164 ; DOI : https://doi.org/ 10.4000/bifea.6164

\section{(c) (i) $\odot$}

Les contenus du Bulletin de l'Institut français d'études andines sont mis à disposition selon les termes de la licence Creative Commons Attribution - Pas d'Utilisation Commerciale - Pas de Modification 4.0 International. 


\title{
LA 'NEGRA NIEVES' OU LE RACISME À FLEUR DE PEAU. REGARDS CROISÉS SUR UNE CARICATURE
}

\author{
Elisabeth CUNIN"
}

\section{Résumé}

Nieves, célèbre caricature colombienne d'une jeune femme 'noire', est l'occasion de mener une réflexion autour de trois questions théoriques : signification des catégories raciales, permanence des stéréotypes et rôle social des apparences physiques. Le personnage, en effet, a une longue histoire : occupant les pages du quotidien de CaliEl País, depuis 1968, il a d'abord été représenté sous forme d'employée domestique avant de devenir, suite à une action de tutelle dénonçant le racisme de la caricature, étudiante en philosophie à l'université. Nieves permet ainsi d'analyser les mécanismes de construction et de gestion de la relation à l'autre : infériorisation paternaliste émanant de l'élite, incorporation populaire de normes sociales racialisantes, ruptures et continuités liées àl'introduction du multiculturalisme, revendications et impasses du mouvement ethnique 'noir', vision culturaliste de l'anthropologie colombienne.

Mots clés Identité, ethnicité, race, racisme, noir, afrocolombien, multiculturalisme, stéréotype.

\section{LA 'NEGRA NIEVES' O EL RACISMO A FLOR DE PIEL. MIRADAS CRUZADAS SOBRE UNA CARICATURA}

\section{Resumen}

Nieves, famosa caricatura colombiana de una mujer 'negra', nos permite hacer una reflexión alrededor de tres cuestiones teóricas: significación de las categorías raciales, permanencia de los estereotipos y papel social de las apariencias físicas. De hecho, el personaje tiene una larga historia: en las páginas del diario de Cali, El País, desde 1968, fue primero representado bajo los rasgos de una empleada doméstica antes de convertirse — en razón de una acción de tutela — en estudiante universitaria de filosofía. Intentaremos así analizar los mecanismos de construcción y manejo de la relación con el otro: inferiorización paternalista por parte de la elite, incorporación popular de las normas raciales, rupturas y continuidades ligadas a la introducción del multiculturalismo, reivindicaciones y dificultades del movimientoétnico 'negro', visión culturalista de la antropología colombiana.

Palabras claves: Identidad, etnicidad, raza, racismo, negro, afrocolombiano, multiculturalismo, estereotipo.

* IFEA-IRD, 32 Avenue Henri Varagnat, 93143 Bondy cedex. E-mail : Elisabeth.Cunin@bondy.ird.fr 


\title{
'BLACK NIEVES' OR RACISM BASED ON APPEARANCES. CROSSED GLANCES ON A CARICATURE
}

\begin{abstract}
In this paper, the use and meaning of Nieves, a famous Colombian caricature of a young 'black' woman, will be explored according to three main theoretical issues: the significance of racial categories, the permanence of stereotypes and the social role of physical appearances. This character has indeed a long history: it first appeared in the pages of the daily newspaper of Cali, El País, in 1968. Before becoming a university philosophy student, the character was initially represented by a domestic employee (the change occurred when the issue of racism was raised and taken to court). The study of Nieves thus makes it possible to analyze how interactions are built and managed: the paternalist pattern of domination emanating from the elite, the widespread use of racial standards, the changes and continuities related to the introduction of the policy of multiculturalism, the ethnic claims and the difficulties of a 'black' movement, the cultural interpretations of Colombian anthropology.
\end{abstract}

Key words: Identity, ethnicity, race, racism, black, afro Colombian, multiculturalism, stereotype.

La caricature de Nieves fut publiée pour la première fois dans le journal El País, quotidien de Cali, le 15 mars 1968 ; depuis, elle a également fait plusieurs apparitions dans d'autres périodiques (El Espectador en 1975 puis de nouveau dans sa version dominicale actuelle, La Prensa et El Colombiano en 1989, Cromos en 1999), des ouvrages lui furent consacrés ainsi que diverses expositions à Cali, Bogotá, Barranquilla, Medellín et même Madrid. Comme le rappelle sa créatrice, Consuelo Lago, membre de l'élite blanche du département du Valle del Cauca, " peu de figures artistiques ont atteint le popularité de Nieves "(Lago, 2001). Mieux même Nieves “ ala capacité d'interpréter tout le pays "(Lago, 2001) et incarne " une conscience collective des thèmes nationaux et des postulats éthiques " (Lago, 2001 : 10). Bref, le personnage créé par Consuelo Lago aurait le pouvoir de révéler le pays à lui-même, de favoriser la réflexion de la société colombienne sur son 'identité nationale'.

Or Nieves, comme son nom ne l'indique pas, est 'noire'. À travers la caricature, l'association entre couleur et statut ne manque pas de surprendre dans un pays qui a traditionnellement relégué ses populations 'noires' à la marge, sociale, politique, culturelle, économique et même géographique de la nation. Si le 'noir' est habituellement perçu comme un citoyen de seconde catégorie, à la fois intégré et discriminé, Nieves occupe, elle, les devants de la scène publique et a la faculté de jouer le rôle de conscience politique nationale. Pourtant, ce 'dilemme de statut' (Hughes, 1994) est immédiatement désamorcé : née à Juanchito, “ à côté du fleuve " (Lago, 2001 :N9ł,ves est aussi employée domestique jusqu'en 1997, date à partir de laquelle, on y reviendra, elle devient étudiante en philosophie à l'université. Les stéréotypes 'raciaux' resurgissent ici, qui réduisent le 'noir' aux habitants des basses terres du Pacifique, humides et chaudes, et l'assignent aux fonctions subalternes, héritées de l'époque esclavagiste. Paradoxe donc que ce personnage de Nieves, à la fois porte-parole national et issue d'un groupe marginalisé, mise en valeur et incarnation du stigmate racial, 'blanche' par son nom et 'noire' par sa couleur. 
La caricature au succès populaire nous permet ainsi d'entrer dans l'analyse de la place accordée au 'noir' dans la société colombienne, entre racisme quotidien qui ne dit pas son nom et paternalisme bien-pensant qui réduit l' autre à un statut inférieur, en passant par sa récupération par le multiculturalisme. Or, l'intérêt du personnage ne se limite pas à la seule expression de la vision de la population 'blanche' (élite, opinion publique, administration) sur le 'noir' ; carNieves a suscité des réactions de la part de nombreux acteurs. En effet, en 1997, une tutelle (1) fut émise par Pascual Charrupí, professeur à l'Universidad del Valle à Cali et représentant de la Fundación para el Avance de la Raza Negra, dénonçant le message raciste de la caricature. Or, si l'on constate que la presse a été unanime à défendre Nieves, tant dans les éditoriaux du principal journal concerné, El País, que dans les lettres envoyées par les lecteurs, il est plus surprenant d'apprendre que les associations 'noires' de l'époque n'ont en rien soutenu l'initiative de Pascual Charrupí. Enfin, comme si cela n'était pas suffisant, Nieves a également fait l'objet d'une autre réappropriation, venue cette fois du champ scientifique. En effet, la revue América Negra, principale instance de diffusion et de convergence des recherches afrocolombianistes dynamisées par la logique multiculturelle née de la Constitution de 1991, a consacré l'un de ses numéros à la jeune employée domestique 'noire'. L'accession de Nieves aux pages d'América Negra ne manque pas d'interroger sur les orientations du discours scientifique, principalement anthropologique. Car loin d'être présentée comme une incarnation du racisme de la société colombienne, Nieves apparaît comme une contribution positive, permettant de sortir les populations 'noires' de l'invisibilité qui les caractériserait.

Le personnage de Nieves est donc bien “ ingénue et irrévérente " (Lago, 2001), non pas tant du fait de ses commentaires sur la réalité nationale, que parce qu'elle incarne, de façon à la fois naïve et subversive, le statut de 'noir', dont la mise en scène quotidienne ne doit pas faire oublier la charge stigmatisante. C'est à une réflexion sur les constructions multiples de la catégorie 'noir' que nous invite Nieves, nous obligeant ainsi à renoncer à toute définition univoque et invariante de l'autre et à prendre pour objet d' analyse non pas une supposée 'identité noire' mais les mécanismes, relationnels, situationnels et partiels, d'attribution de statut.

Je m'intéresserai moins ici au contenu des images elles-mêmes qu'à leur perception par de multiples acteurs ; une telle approche peut sembler paradoxale au moment même où la réflexion porte sur le poids des apparences dans la désignation de l'autre. Mais il s'agit de s'interroger sur l'interprétation - et, plus exactement, sur la multiplicité des interprétations — des images plus que sur les images elles-mêmes. Délaissant ainsi l'analyse de l'évolution de la représentation de Nieves, des différents personnages qui l'entourent, des scènes et mises en contexte (qui pourrait faire l'objet d'un autre article), je centrerai mon attention sur le regard plus que sur l'objet du regard, sur les signifiés plus que sur le signifiant lui-même, afin de saisir les mécanismes sociaux de construction polyphonique et polysémique de l'autre racial (2).

(1) Mécanisme juridique introduit par la Constitution de 1991 permettant une interpellation directe, par les citoyens, de la justice.

(2) Une autre nuance méthodologique doit être apportée : seule la dimension raciale de la caricature est étudiée ici, alors qu'elle aurait nécessité une analyse mettant également en lumière les ressorts de la construction de la catégorie 'femme' et la mobilisation de traits identitaires 'sexualisants', venant se superposer aux traits racialisants. 


\section{QUELQUES ENJEUX THÉORIQUES}

La caricature et son acception différentielle mettent en jeu toute l'ambiguïté du statut du 'noir' ; le personnage deNieves ouvre à une réflexion sur la permanence des identifications raciales dans une société qui, depuis qu' elle s'est déclarée pluriculturelle (Constitution de 1991) tend à substituer l'ethnicité à la race, la culture à la nature, l'afrocolombien au 'noir'. Aussi est-on également amené à s'interroger sur le rôle des stéréotypes, ces images préconstruites qui agissent comme un répertoire a priori permettant de classer l' autre, et à en appeler à une véritable sociologie des apparences, tant ce qui est immédiatement visible (ici, la couleur) devient marqueur d'identité.

\section{1. Autour de la notion de race}

Une précaution préalable s'impose du fait du tabou associé, plus particulièrement en Europe, à l'utilisation du terme race, précaution qui nous invite, au-delà de cette seule catégorie polémique, à réfléchir sur les concepts utilisés par le chercheur. Le point de départ de cet article doit être posé très clairement et fermement : les races n' existent pas. Plus exactement, le terme renvoie à un objet qui n'existe pas mais qui devient réalité par le seul fait de sa désignation, donnant naissance à des pratiques sociales qui, elles, existent bien (Guillaumin, 1992). La race sera donc considérée ici non comme une catégorie analytique, mais comme une catégorie pratique, sociale et politique, déterminée par l'idée de l'existence présumée de races (Brubaker, 2001). D'une part, la démonstration - qu'elle provienne des sciences sociales ou des sciences naturelles — de l'inexactitude du terme ne suffit pas à l'effacer des activités sociales, tant cognitives que pratiques ; d'autre part, la confusion entre acceptions populaire et scientifique constitue une dimension intrinsèque de cette catégorie (Wacquant, 1997), avec laquelle il s'agit désormais de se distancier de façon explicite. Le chercheur est dans l'impossibilité d'appréhender la race comme un attribut des individus ou des groupes, qui pourrait être découvert, décrit et défini : la race est une catégorie populaire à travers laquelle est déchiffré et interprété l'environnement social, passé et présent, qui donne sens aux pratiques quotidiennes et permet d'évaluer et de classer l'autre. Seuls les processus sociaux à travers lesquels sont produites, connues et adoptées les catégories pratiques constituent un objet pour une recherche qui ne se place pas seulement du côté du constructivisme (fluidité, multiplicité des identités) mais qui vise également à comprendre les mécanismes de réification des pratiques identitaires (3). Face aux analyses de la race, del'ethnicité et de l'identité, qui ont inspiré les premiers travaux colombiens naturalisant l'altérité, qu'elle soit définie en termes physiques ou culturels, il s'agit de s'intéresser aux mécanismes, dynamiques et interactionnels, d'élaboration et de consolidation des frontières entre 'eux' et 'nous' et, au-delà, d'émergence et de fixation des normes sociales.

(3) “ Nous soutenons que l'approche constructiviste de l'identité qui prévaut actuellement - la tentative d' 'adoucir' le terme et de lever l'accusation d' 'essentialisme' qui pèse sur lui en stipulant que les identités sont construites, fluides et multiples - ne justifie plus que l'on parle d' 'identités' et nous prive des outils nécessaires à l' examen de la dynamique 'dure' et des revendications essentialistes des politiques identitaires contemporaines " (Brubaker, 2001 : 66). 
La tradition française écrit le mot race avec des guillemets, afin d'affirmer la distance prise vis-à-vis du terme. Or, si les guillemets tendent à rendre visible la distinction entre catégories pratique et analytique, on peut alors se demander pourquoi leur usage n'est pas généralisé, étendu à des termes qui, comme l'identité, l'ethnicité, la nation, sont aujourd' hui fortement contestés au sein du champ scientifique alors qu'ils sont de plus en plus utilisés par les acteurs eux-mêmes. Sans doute la mention de la race, en raison de l'usage dramatique qui en a été fait et du rejet catégorique qui l'a suivi (venant par exemple de l'UNESCO) rend-elle ces questions plus polémiques et délicates ; pourtant les réflexions sur la race s'inscrivent dans la même logique que celles portant sur d'autres catégories d'appartenance. Les travaux sur l'identité, la nation ou la race étudient désormais les modes de construction et de réification de ces notions à partir des logiques d' action des individus et des groupes (4). Ce sont les formes sociales d'existence de ces appartenances qui nous intéresseront ; c'est pourquoi je n'utiliserai pas les guillemets autour des mots race, identité, ethnicité, etc., mais j'insiste sur le fait qu'il s'agit de catégories pratiques d'appartenance et non de catégories scientifiques.

De même, je ne céderai pas à l'usage courant qui transforme une apparence approximative et socialement construite en catégorie d'appartenance pour laquelle le substantif, rendu à l'écrit par la majuscule, renvoie à l'idée d'une identité qui serait 'naturelle' et allant de soi. Contre cette écriture consensuelle et politiquement correcte, j'emploierai les termes 'noir', 'blanc' et leurs dérivés ('métis', 'mulâtre', 'moreno', etc.) sans majuscule mais entre guillemets. Ces désignations constituent des catégories opérationnelles de l'action, des classifications sociales mobilisées en situation et renvoyant à des savoirs et des normes diffus et implicites. Alors que la division entre les races est présentée comme une dimension naturelle de l'ordre social, je souhaite montrer qu'elle est avant tout un construit social, porteur de normes et de valeurs. Les différences raciales sont inscrites dans une nature biologique qui est elle-même une construction sociale naturalisée et naturalisante. En ce sens, la démarche adoptée ici est, de façon indistincte, une déconstruction del'objectivation raciale et une mise en cause des formes de la connaissance. De même que dans les rapports entre les sexes (Bourdieu, 1998), les relations raciales reposent sur une causalité circulaire qui inscrit les différences culturelles dans la 'nature' des individus, qui fait de la différence physique, socialement construite, le fondement 'naturel' de divisions perçues comme objectives, car immédiatement visibles, et subjectives, car intégrées aux schèmes cognitifs.

\section{2. Permanence et fonction des stéréotypes}

On définira le stéréotype comme la réduction d'une identité ou d'une représentation à quelques traits simplifiés, aux connotations négatives ou, au moins, infériorisantes, obéissant à une logique essentialisante (les éléments d'identification ne sont liés ni au contexte, ni à l'histoire, ni aux interactions) et catégorielle (les individus appartenant à une catégorie étant immédiatement dotés de tous les attributs définissant cette catégorie)

(4) Avec, néanmoins, une différence de taille : la notion de race se construit en permanence sur une relation ambiguë entre le biologique et le culturel (Bonniol, 1992). 
(5). La caricature de Nieves s'inscrit bien dans ce cadre : elle présente un personnage ayant un statut doublement subalterne (servante et 'noire'), dont les traits physiques (couleur, cheveux), psychologiques (naïveté, bonne humeur, simplicité) et culturels (langage, mode vestimentaire) sont immuables (6) et symbolisent la 'race noire' dans son ensemble.

Le stéréotype a deux fonctions principales : d'abord une fonction identitaire en permettant le maintien de la cohésion d' un groupe à partir de l'identification de ceux qui lui sont extérieurs. La réitération d'un certain nombre de traits (physiques, culturels, psychologiques) permet l'affirmation de la différence et la réactivation de la frontière entre 'nous' et 'eux'. On est ici dans un double processus d'identification / altérisation et inclusion / exclusion. En ce sens, le 'noir' (et l' 'indien') est une catégorie historique permettant la construction d'une identité nationale qui s'inscrit en négatif : 'blanc' /

'noir', intérieur / côte, terres hautes / terres basses, climat froid / climat chaud et, audelà, civilisation / sauvagerie, esprit / corps, éducation / instincts, etc. Aussi bien, le stéréotype nous informe-t-il moins sur l'objet qu'il est censé décrire, que sur le groupe producteur lui-même, sa conception de l'identité et de l'altérité. Plus les groupes sont proches (en ville par exemple), plus le danger de 'contamination' est grand, plus le stéréotype sera marqué et plus il est facilement partageable et mobilisable.

Le stéréotype a également une fonction que l'on pourrait qualifier de communicationnelle : il favorise la communication en évitant le rappel d'implicites partagés. On ne redéfinit pas les règles du jeu à chaque fois que l'on commence une nouvelle partie, mais on fonctionne bien plus, par économie de la pensée, sur un accord considéré comme préalable à l'interaction (c'est le " cadre de l'expérience " de Goffman). Le locuteur fait comme si son interlocuteur partageait un certain nombre de savoirs déjà assimilés qui autorisent l'existence d'un “ sens commun " ou d'un " lieu commun " ainsi que l'entend Glissant (1997 :23)

“ Combien de personnes en même temps, sous des auspices contraires ou convergents, pensent les mêmes choses, posent les mêmes questions. Tout est dans tout, sans s'y confondre par force (...). C'est ce qui désigne le lieu commun. Il rameute, mieux qu'aucun système d'idées, nos imaginaires "

Le stéréotype agit comme un véritable moule dans lequel s'inscrit la perception de l'autre, une “ matrice d'opinion "plus qu' une opinion (Boëtsch \& Villain-Gandossi, 2001 : 19), qui se situerait en amont et non en aval des représentations. Le jugement (ou le préjugé) précède l'expérience. Le stéréotype n'est jamais plus efficace que lorsqu'il n'apparaît pas comme un stéréotype mais comme une norme de comportement. C'est bien pourquoi il est si difficile de s'en défaire et même d'en prendre conscience ; il alimente ainsi un racisme diffus, anodin parce que quotidien, invisible parce qu' ordinaire, impensé parce que naturalisé. Ce n'est pas seulement le racisme qui est pernicieux mais le stéréotype qui l'accompagne et le précède même, cette sorte de " racisme avant le racisme "(Villain-Gandossi, 2001 : 32) qui n'est donc pas remis en cause lorsque le racisme lui-même est condamné.

(5) On retrouve des approches similaires dans certains travaux contemporains qualifiés de 'post-modernistes' ou de 'cultural studies' : voir la “ cryométonymie ” de Appadurai (2001) ou la critique de l'orientalisme de Said (1978).

(6) Depuis sa naissance, la représentation du personnage de Nieves n'a que très peu évolué. 
On voitici la logique d'action des stéréotypes : ils se présentent comme une vérité incontournable, ils sont les significations elles-mêmes. L'individu n'est pas un sujet mais un signe, et ce signe envoie sur celui qui le porte tout un faisceau de 'connaissances' incorporées. Le stéréotype fonctionne alors par association d'idées : un mot, une expression, une image réfèrent à un halo de connotations, d'autant plus efficaces qu'elles sont confuses et implicites. La richesse et la force des stéréotypes, c' est qu'ils sont à la fois extrêmement rigides, fixes, immobiles, répétitifs et constamment mobilisés dans des contextes différents, adaptables aux situations et aux interlocuteurs. Ils présentent à la fois une grande résistance au changement car ils sont indépendants de l'expérience (on trouve, par exemple, dans les textes de José Francisco de Caldas, au tout début du $19{ }^{\text {ème }}$ siècle, les même représentations naturalisantes et infériorisantes du 'noir' que celles qui s' expriment aujourd' hui dans la qualification - et parfois la valorisation d'une musique 'noire' comme la champeta ou de la première reine de beauté nationale 'noire') et un mode d'expression sans cesse renouvelé. Avec la caricature, le physique, les apparences, à la fois objectifs et subjectifs, de l'ordre du visuel et du signe, génèrent des qualifications mentales et culturelles, manipulées et naturalisées. Le stéréotype masque alors tout en démasquant celui qui le produit, forme tout en déformant.

\section{3. Pour une sociologie des apparences}

Consuelo Lago donne à la couleur de son personnage un statut purement esthétique : dans un journal dont les pages sont blanches, le 'noir' est évidemment immédiatement visible. L'auteur rappelle d'ailleurs l'anecdote selon laquelle la première caricature de Nieves proposée à l'imprimerie du journal aurait été 'blanche' ; c'est en constatant le peu de visibilité d'un personnage 'blanc' que l'artiste l'aurait peinte en 'noir'. Nieves est ainsi décrite comme " une tâche noire sur un papier blanc, pour attirer le regard. Une femme blanche, on ne la regarderait pas autant. Si le papier avait été noir, je l'aurais faite blanche. C'est une question esthétique "Entretien, 14 juin 2002).

Dans un pays où la notion d' 'invisibilité' est mobilisée par les anthropologues pour évoquer le statut des populations 'noires' (Friedemann, 1993), on ne peut donc que s'interroger sur les ambiguïtés d'un concept qualifiant d'invisibles des individus qui se distinguent précisément par leur différence physique, dont la couleur, immédiatement perceptible, rompt avec un modèle idéal de blancheur, plus ou moins ouvertement accepté. Cette contradiction entre invisibilité scientifique et visibilité corporelle amène à s'interroger sur les éléments pertinents d'identification de l'autre et à prendre au sérieux les mécanismes de fonctionnement du regard. Il n'est pas inutile de confronter directement les propos de Nina de Friedemann, dont les premiers travaux vont profondément influencer le développement ultérieur des recherches afrocolombiennes, aux recommandations d'Erving Goffman. Nina de Friedemann appelle en effet l'anthropologie à aller chercher la 'vérité' au delà des apparences : “ Le défi des études afroaméricaines est de saisir, non pas le masque, mais celui qui porte le masque " (Friedemann, 1993 : 170). Au contraire, pour Goffman, “ la nature la plus profonde de l'individu est à fleur de peau " (Goffman, 1973 :338). Il faut donc s'intéresser à la façon dont les individus perçoivent leurs apparences mutuelles, en combinant évaluation individuelle et mobilisation des normes sociales. Des imprécisions de ces processus, les 
individus tirent leur capacité à modifier leur présentation, à négocier les attentes réciproques, à s'adapter à une situation particulière. Les traits raciaux constituent autant d'éléments de répertoire pour l'individu et autant de facteurs de décision pour les autres, qui s'efforcent de lui assigner un statut. Il ne s'agit donc pas d'aller chercher la vérité au-delà de ce qui se voit, mais de découvrir la profondeur de la superficialité, en s'intéressant aux apparences dont la gestion repose sur la capacité des individus à qualifier la situation, à répondre aux attentes et à se positionner soi-même, à interpréter les conventions de la rencontre et à mobiliser un savoir socio-historique souvent diffus et implicite.

Car, à travers le personnage de Nieves, 'ce qui est vu' nous informe sur les formes pratiques d'évaluation et d'interprétation d'une situation (7) et 'ce qui est donné à voir' sur les principes qui régissent les normes sociales et ordonnent le sens commun. La primauté rendue au rôle joué par les apparences ouvre ainsi la voie à une approche territoriale : dans la présentation et la représentation du corps s'expriment et se façonnent une identité individuelle et des normes sociales. 'Donnée objective' qui agit comme un facteur d'identification déterminant un certain type d'appartenance (raciale, ethnique et, au-delà, sociale), la couleur est aussi une catégorie subjective, socialement construite, dont la signification et la saillance varient avec les individus et les situations. Ce processus circulaire de l'objet au sujet et du sujet à l'objet, ces apparences à la fois déterminées et déterminantes, renvoient aux mécanismes cognitifs de désignation de l'environnement physique, aux formes de la perception du monde, au " sens d'être du monde "qui ne présupposent “ ni l'idée naïve de l'être en soi, ni l'idée, corrélative, d'un être de représentation, d'un être pour la conscience, d'un être pour l'homme "(MerleauPonty, 1964 : 21).

Si la tendance actuelle est à diagnostiquer un " racisme sans race " (Balibar \& Wallerstein, 1990) ou un " néoracisme culturel " (Chebel, 1998), dans lequel le thème dominant ne serait plus l'altérité biologique, mais l'irréductibilité des différences culturelles, la couleur est précisément un de ces construits culturels qui interviennent dans l'assignation d'un individu à telle catégorie d'appartenance. Opposer un racisme biologique et un racisme culturel revient, dans une certaine mesure, à faire de la couleur un marqueur d'identité 'naturel', alors qu' elle est, comme toute différence socialement construite, un signe social donnant lieu à des interprétations multiples, manipulable dans des stratégies de classement social, d'attribution de statut à l'autre et de présentation de soi. Loin de se superposer systématiquement, apparences et appartenances entretiennent une relation dynamique et processuelle, dans un mouvement d'identification circulaire. Mon intention, à travers l'étude de la caricature de Nieves, est précisément de prendre au sérieux le rôle des apparences dans l'interprétation d'une situation et dans l'attribution de statuts.

Ici, les apparences, mises en scène, fonctionnent comme un marqueur d'identité : on passe ainsi des traits physiques aux caractéristiques raciales puis, des caractéristiques raciales, un nouveau pallier est franchi vers le groupe racial. Sous les traits physiques représentés sont introduites des propriétés d'un autre ordre, sociales et culturelles, qui

(7) “ La compétence sociale de l'œil est énorme ”(Goffman, 1988 : 153). 
qualifieraient un ensemble homogène d'individus, les 'noirs'. Car l'image n'a pas seulement un pouvoir descriptif : elle est également prescriptive, elle définit une réalité qui doit être selon certaines normes. Dans le même temps, ce glissement s'opère sur le mode du 'vu non-dit' ; l'esthétique des normes a d'autant plus de portée qu'elle repose sur un enchaînement logique implicite : perception visuelle évidente-donc-communedonc-normale. Le 'vu' agit comme une garantie qui fonde le partage avec autrui d'une même impression et, au-delà, l' assurance de bien comprendre la même chose ensemble. Alors même que le 'visible' est, ici comme ailleurs, le fruit d'une production sociale résultant de l'imposition d'une interprétation dominante de l'histoire et du présent des relations à l'autre. Les apparences esthétisées sont à la fois du domaine de l'évidence visuelle et de l'image construite, l'immédiateté du monde commun et l'artificialité de la mise en scène se superposant alors.

\section{UN RÉVÉLATEUR DES RELATIONS À L’AUTRE}

Nieves, comme de nombreuses autres caricatures populaires en Amérique Latine, incarne l'idée populiste du peuple sage, capable de voir et de dire ce que les autres ne pourraient pas. Clairvoyance populaire qui, bien entendu, ne peut être énoncée que par l'élite elle-même, dans une logique de légitimation et renforcement de l'ordre social existant, de normalisation des règles sociales ordinaires. Je m'intéresserai donc tout d'abord au pouvoir révélateur du personnage de Nieves : révélateur du paternalisme racisant de l'élite en premier lieu, révélateur du racisme quotidien de l'opinion publique ensuite, révélateur, enfin, de l'instrumentalisation actuelle de la différence par le multiculturalisme. On verra ainsi à quel point les catégories raciales et l'organisation socio-raciale sont incorporées, fonctionnant comme un véritable habitus structurant les pratiques et les discours.

\section{1. "Les noirs me semblent beaux et drôles. Je suis de leur côté " (Consuelo Lago, El País, 2 septembre 1997)}

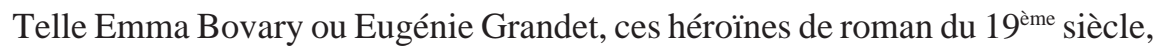
Consuelo Lago s'ennuyait dans son rôle de bourgeoise provinciale, préférant renoncer au bureau doré de la direction d'une entreprise pour suivre une carrière artistique, correspondant davantage à des aspirations bohèmes et aventurières facilitées par l'appartenance à l'élite de la société colombienne. C'est ainsi que naîtra Nieves (8), transformant l'oisiveté de sa créatrice en mission sociale. Car Consuelo Lago se fait un honneur de contribuer, grâce à Nieves, à plusieurs institutions de bienfaisance, du Club Noel à la Cruz Roja en passant par le Banco de Ojos ou l'Instituto para Niños Ciegos y Sordos de Cali. Évoquant les raisons qui l'ont poussée à se consacrer à un personnage 'noir', Consuelo Lago témoigne du paternalisme bien-pensant, héritier des pères jésuites Alonso de Sandoval et Pedro Claver, idéologie de l'harmonie raciale dans laquelle chacun, 'blanc' et 'noir', occupe une position bien définie, qui marque encore

(8) Rappelons que le personnage de Nieves faillit bien ne jamais voir le jour : la première intention de Consuelo Lago fut d'écrire un roman, dont un épisode aurait été publié quotidiennement dans les pages du journal de Cali. 
aujourd'hui le discours de l'élite colombienne. “ Il y a toujours eu beaucoup de population 'noire' à Cali, culturellement je suis habituée à ce que les 'noirs' soient partout. Il y a toujours eu une employée "noire' dans ma maison "Entretien, 14 juin 2002) (9). Et si le 'blanc' peut vaquer en toute bonne conscience à ses activités artistiques et de bienfaisance, le 'noir' est quant à lui réduit aux fonctions serviles et à un rôle subalterne. Sans que cette assignation à résidence identitaire, infériorisante et stigmatisante, ne soit perçue comme problématique. De fait, c'est précisément parce que les statuts sont définis de façon formelle que l'autre ne dérange pas, que le face-à-face n'est pas embarrassant. Ainsi, dans un surprenant mélange de naïveté et de cynisme, le 'noir' est-il à la fois accepté et rejeté, proche et lointain. Comme si sa seule valeur était de servir, autrefois esclave, aujourd'hui employée domestique, il semble naturel à Consuelo Lago que Nieves, en tant que personne 'noire', doive consacrer sa vie à lui venir en aide. C'est précisément ainsi que Nieves est née : “ j’avais besoin que quelqu'un m'aide à travailler et les personnes qui m'ont toujours aidée sont 'noires' ".

Si toute accusation de discrimination ou de mise à l'écart est désamorcée avant même d'être menaçante dans cette idéologie humanitariste, l'autre est réduit à une place limitée et prédéfinie, n'échappant pas à la mobilisation des stéréotypes qui accompagnent la description du 'noir' depuis l'époque coloniale et qui marquent les discours sur l'identité nationale. Dans sa première apparition (15 mars 1968), Nieves utilise un langage qui rend compte tout à la fois du respect exprimé vis-à-vis de ses employeurs et des déformations imprimées au vocabulaire correct : “ je vais voter pour M'dame soffy et M'dame leonor. Je ne vote pour aucun M'sieur " ‘o voy a votar por misiá soffy y por misiá leonor. Yo no voto por ningun senó). D’ailleurs, le nom du compagnon de Nieves témoigne lui aussi de cette prononciation qui serait propre, selon Consuelo Lago, aux individus 'noirs' : “ son nom est Hector mais les gens 'noirs' disent Hétor ”. La naturalisation de la différence s'étend à l'association, posée comme évidente, entre le 'noir' et un environnement physique exubérant, caractérisé par un climat chaud et des terres basses. Évoquant le sort des migrants du Pacifique à Bogotá (déplacés par la violence ou par la pauvreté), Consuelo Lago ne manque pas de s'apitoyer sur leur sort : “ ce qui me fait de la peine ici [à Bogotá, où Consuelo Lago s'est installée depuis quelques années] c'est que ce qu'ils aiment c'est la chaleur, ici ce n'est pas un climat qui leur convient. Les 'noirs' aiment vivre à côté du fleuve ou de la mer "(10).

Consuelo Lago, originaire de Cali, nous transmet donc la vision caractéristique de l'élite vallecaucana et, au-delà, le discours dominant sur l'autre. Il n'est pas inutile de rapporter ses propos au lendemain de la tutelle émise contre son personnage. Selon elle, Nieves, loin d'être l'incarnation du stigmate racial, œuvre en faveur de la reconnaissance des populations 'noires' : “ dans mon cas, la tutelle fut quelque chose d'injuste. Je ne considère pas que Nieves ait discrédité les gens 'noirs', mais bien au contraire qu'elle a aidé la communauté. Nieves ne fait pas de mal mais du bien ". La défense de son personnage suit deux voies caractéristiques du regard dominant sur les

(9) Les citations de Consuelo Lago qui suivent sont issues de cet entretien.

(10) Précisons d'ailleurs que l'œuvre de Consuelo Lago ne se limite pas aux caricatures de Nieves : elle inclut également des peintures, d'inspiration naïve, représentant une nature tropicale, dans laquelle apparaît parfois un personnage 'noir' sur un canoë. 


\section{Productividad es... Acasanumae BOGOTA D.C.}

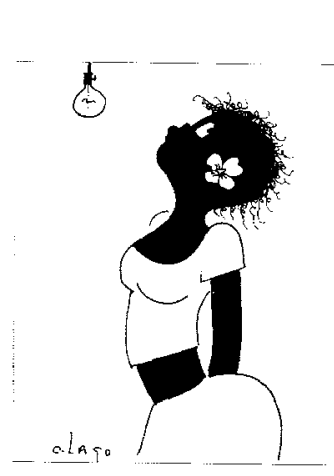

Llamar al electricista en lugar de electrocutarse

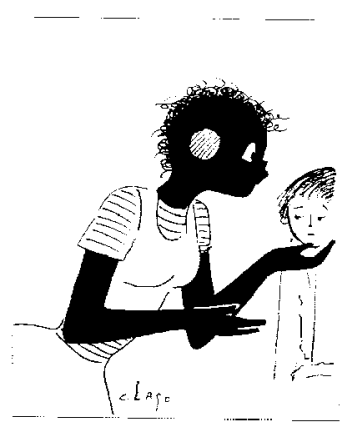

No copiarle el examen al vecino. No hacer trampa

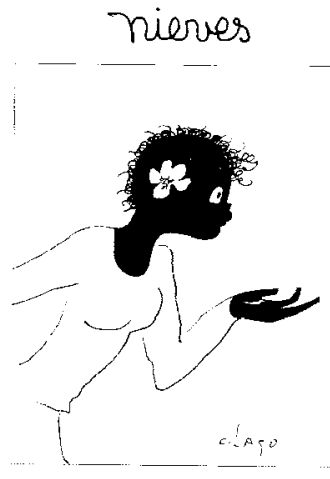

Ser parte de la solución. no del problema

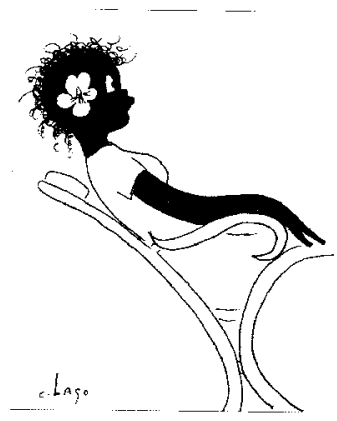

Descansar y volver a empezar

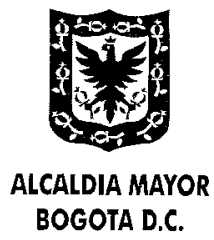

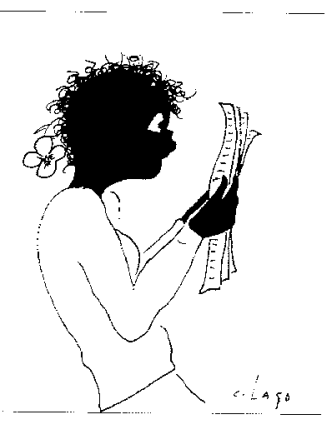

Leer las instrucciones

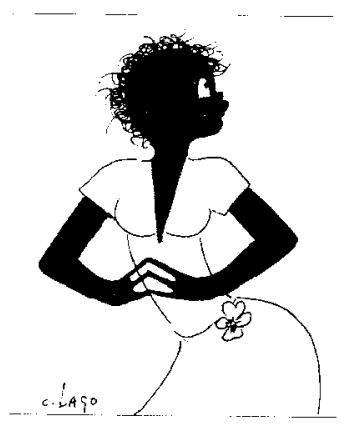

Saber lo que se necesita y alistarlo todo

Fig. 1 - Campagne de production de la productivité organisée par la Mairie de Bogotá. 

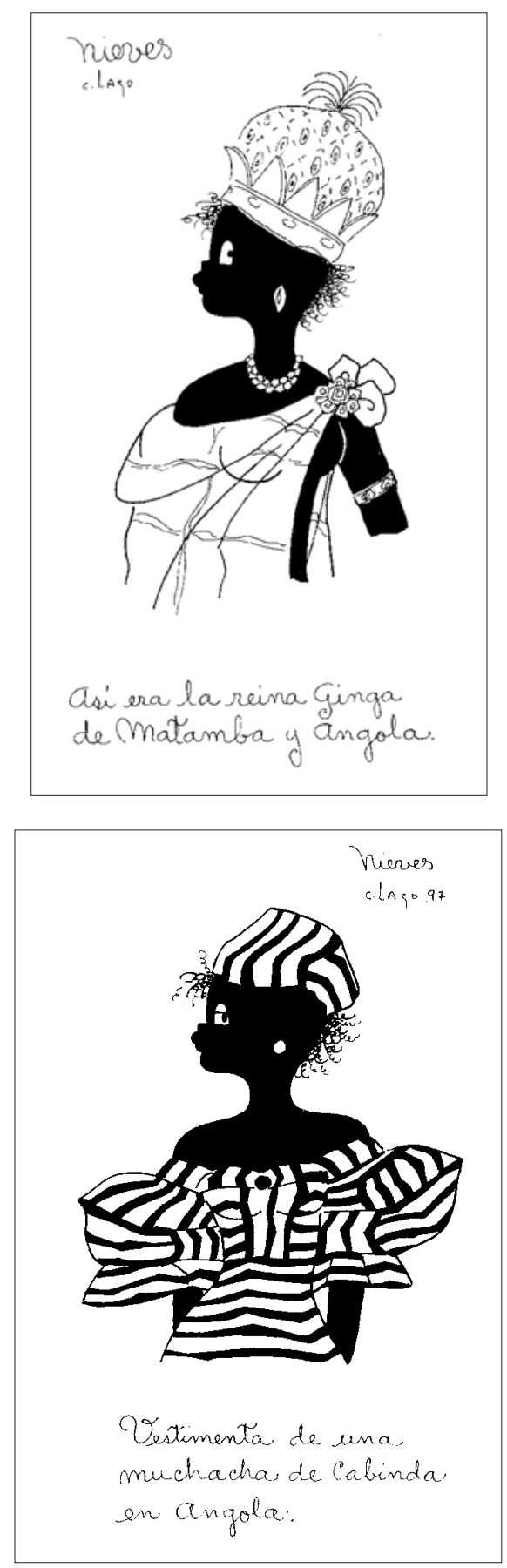

Figs. 2 et 3 - América Negra, nº 12, décembre 1996. 

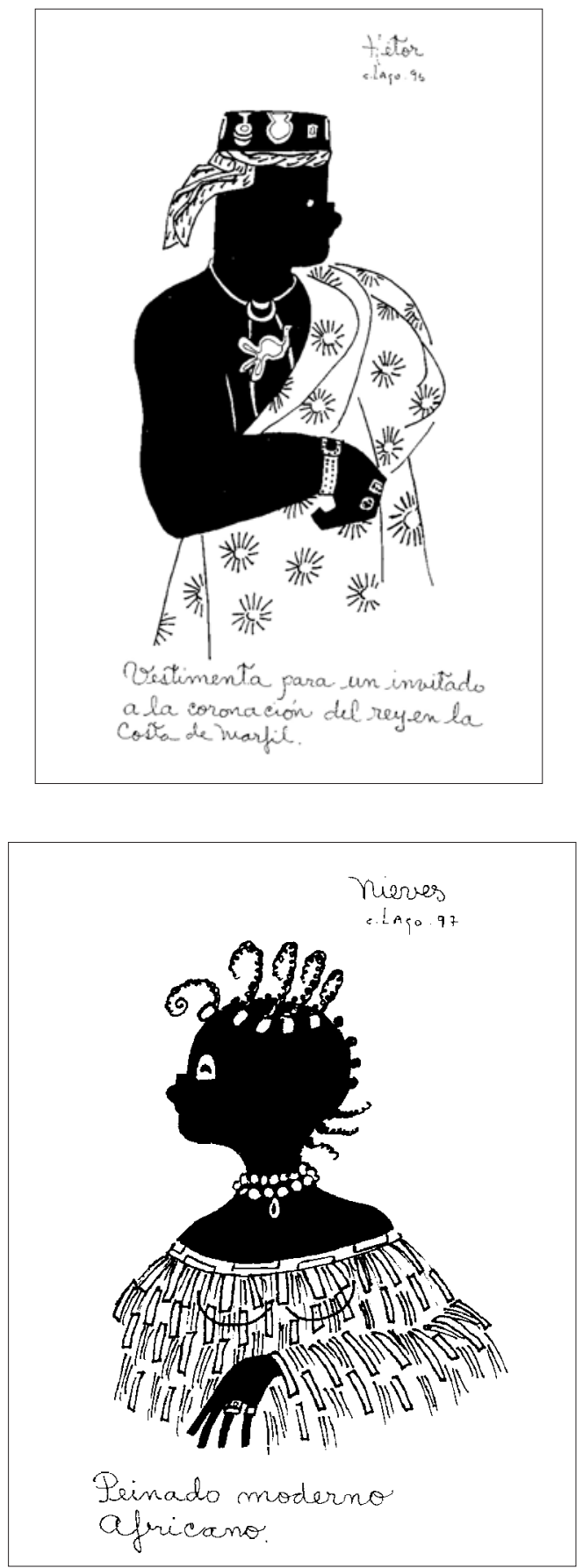

Figs. 2 et 3 - América Negra, no 12, décembre 1996. 


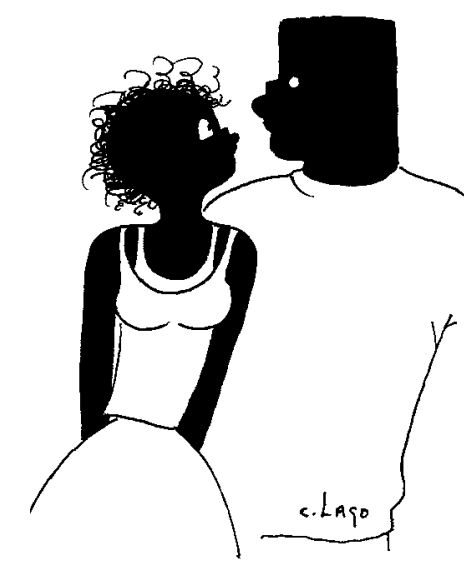

SÍ, HÉTOR. ESTÁ ESCRITO QUE LO ÚNICO QUE TÚ Y YO TENEMOS EN COMÚN, ES LA DIFERENCLA DE SEXO.

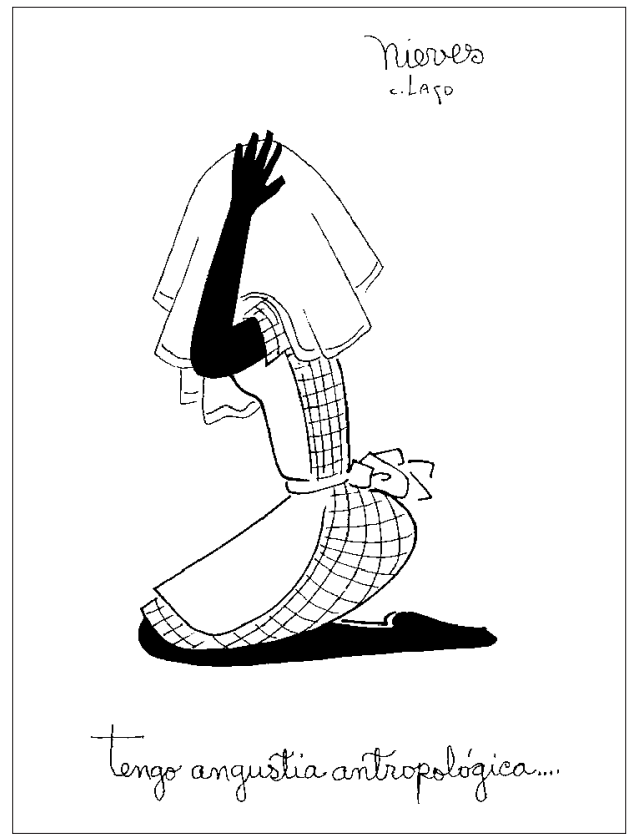

Figs. 4 et 5 - Consuelo Lago, Nieves impertinente et coqueta, Bogotá, Villegas Editores, 2000. 

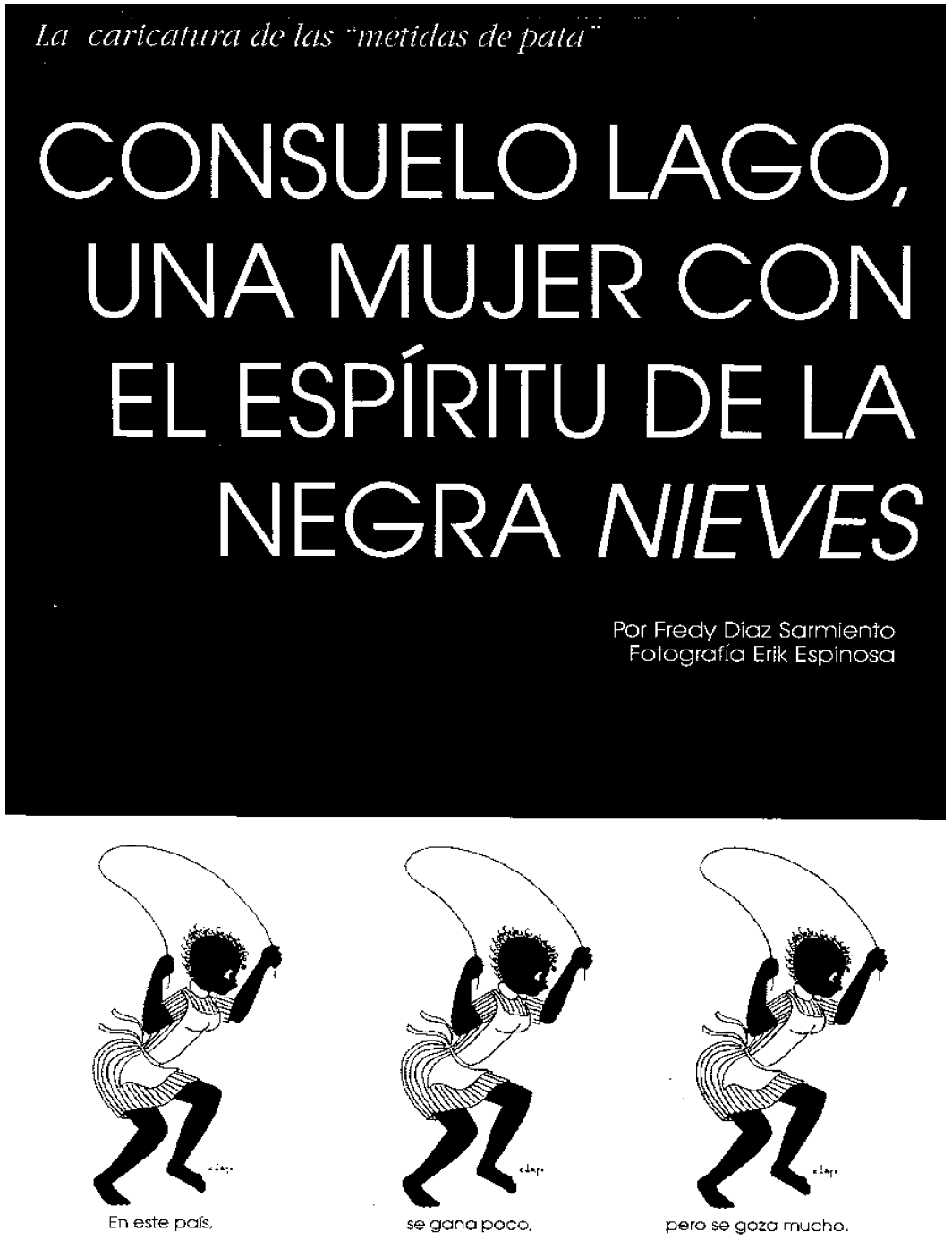

AGENDA CLILTURAL

4

Fig. 6 - Agenda Cultural, Universidad Jorge Tadeo Lozano, nº 136, juin 2002. 
populations 'noires'. Tout d'abord, Consuelo Lago rappelle qu'elle apprécie les 'noirs', qu'elle les a toujours côtoyés dans la maison de son enfance, qu'ils sont pour elle des individus familiers et proches. Comment pourrait-elle alors faire preuve de discrimination à leur égard, comme le prétend la tutelle ?'Nieves et Hétor sont noirs parce que les noirs me semblent beaux. J'ai toujours admiré leur beauté physique et leur grâce, leur sveltesse et leur aisance "El País, 6 septembre 1997). Bref, black is beautiful dans le langage bien-pensant de l'élite, surtout lorsqu'il est réduit à quelques traits physiques (pointant eux-mêmes vers des caractéristiques sociales et culturelles) et à une fonction subalterne. Pourtant, alors même que Nieves est 'noire' (physiquement et du fait de sa naissance dans le Pacifique), la dimension raciale n'est jamais évoquée dans les textes accompagnant les caricatures, comme s'il existait une forme d'évitement de l'identification explicite raciale, comme si les marqueurs raciaux étaient écartés dans la description de l'autre. Dès lors, l'association visuelle entre la couleur et le statut transforme le non-dit verbal en évidence visuelle, qui devient à son tour normative (ce qu'est le 'noir') et performative (ce que doit être le 'noir').

Et lorsque la dimension raciale est directement mobilisée, comme ce fut le cas avec la tutelle, sa signification est immédiatement neutralisée et euphémisée, lui ôtant toute pertinence en tant qu'élément d'attribution de statut. “ Le fait qu'elle fasse des tâches ménagères et que son compagnon, Hétor, n'ait pas de travail permanent ne signifie pas que je sois en train de déprécier une race en particulier, parce que dans toutes les races il y a des personnages comme cela "El País, 6 septembre 1997). D'ailleurs, comme le rappelle Consuelo Lago, les amies de Nieves ne sont-elles pas servantes elles aussi, mais 'blanches' ? Alors même que la caricature fonctionne sur un principe de catégorisation - les individus appartenant à la catégorie incarnée parNieves étant immédiatement dotés des mêmes caractéristiques —- Consuelo Lago se situe dans une logique d'individualisation, désamorçant ainsi par son discours la portée de ses dessins.

Gage de sa grandeur d'âme, Consuelo Lago a supprimé, suite à la tutelle de Pascual Charrupí, le tablier qui accompagnait Nieves avant 1997. “ Je lui ai enlevé son tablier pour ne faire de mal à personne. Pour moi, la tutelle était contre le tablier. Si le tablier offensait la race 'noire', je l' ai enlevé parce que je ne veux pas blesser. Je l'ai mise à l'université, pour étudier la philosophie. Maintenant elle peut continuer à dire les mêmes choses, mais sans tablier. Et ses amies sont toujours 'blanches', mais avec un tablier '”. Tour de passe-passe symbolique, la substitution de l'habit d'étudiante à celui de servante suffirait à éliminer toute accusation de racisme. On est ici dans une logique d'inversion du stigmate, d'utilisation de désidentificateurs (les études) qui permettraient de compenser les identificateurs raciaux (la couleur). Mais ceux-ci ne sont absolument pas remis en cause, les normes associées au 'noir' n'ont pas été affectées. Dans le mouvement même de valorisation du personnage de Nieves sont finalement réaffirmées les normes sociales associées à la catégorie raciale. On voit ainsi émerger l'ambiguïté du discours de Consuelo Lago. D'une part, elle témoigne d'un certain mépris pour cette transformation, considérée comme une concession inutile et injustifiée à une idéologie inspirée du 'politiquement correct' : le fait queVieves soit servante n' a rien à voir avec son appartenance raciale. Mais, d' autre part, les caricatures qui suivent la tutelle rendent compte du caractère anormal du nouveau statut de Nieves. Ses collègues employées 
domestiques s'interrogent : 'Nieves va devoir travailler le jour et étudier la médecine la nuit (11). Que va dire Hétor ? El País, 19 septembre 1997) et Nieves elle-même doit faire un effort pour entrer dans son nouveau rôle : " Je suis enfermée à étudier la philosophie "El País, 21 septembre 1997). Présenté comme étant insignifiant, le marqueur 'racial' est à tel point déterminant qu'il pèse même lorsqu'il est supposé avoir disparu, alors que l' association naturalisée entre ‘noire' et 'servante' n'est pas interrogée.

\section{2. "En défense de la 'negrita' 'El País, 6 septembre 1997)}

La caricature de Nieves invite à s'intéresser aux formes de gestion ordinaire des catégories raciales, au racisme quotidien (Essed, 1991), implicite, anodin, minoré parce que reposant sur le regard, oublié parce que normalisé. L'invisibilité croît à mesure que l'individu s'inscrit dans l' 'ordre des choses', elle se renforce avec la 'normalité'. En ce sens, paradoxalement, Nieves semble bien être invisible. Mais cette invisibilité, loin de devoir être dépassée et inversée (comme le réclame le nouveau discours afrocolombien), appelle plutôt une réflexion sur les mécanismes sociaux de neutralisation des apparences raciales, sur le mode de fonctionnement du vu et du visible.

Suite à la tutelle émise par Pascual Charrupí, le juge chargé du dossier a émis un avis défavorable, rejetant toute accusation de racisme. Non seulement le personnage de Nieves n'est pas porteur d'une vision discriminante de l'autre, mais il autorise au contraire la mise en valeur d'une minorité généralement marginalisée. Mieux même : la défense du juge n'hésite pas à reprendre les stéréotypes associés au 'noir', en leur donnant cette fois une signification positive.

“ On trouve dans cette caricature le mode de vie charismatique de notre population simple, modeste, sincère, franche : avec sa façon extrovertie et simple de voir les choses, quelle que soit sa couleur (...). La caricature de Nieves ne dit absolument rien contre la race noire, bien au contraire, elle la glorifie pour son esprit, sa malice et son intelligence (...). Il faut insister sur l'œuvre sociale que constitue la caricature et son auteure, la caricature de Nieves fait plus de bien que de mal et, plus que d'affecter les droits des noirs, elle glorifie leur race ".

De même, le courrier des lecteurs d'El País s'est fait l'écho des réactions de l'opinion : toutes ont pris la défense de Consuelo Lago et deNieves. L'initiative de Pascual Charrupín'est pas comprise : “ C'est incroyable qu'un homme si lettré, comme je suppose que l'est Pascual Augusto Charrupí, affirme qu'il ira jusqu' au bout en voyant son action de tutelle rejetée "El País, 6 septembre 1997) ; la dimension raciale de la caricature est évacuée : “ je crois qu'ils ont exagéré parce que ce que dit cette sympathique jeune fille n'a aucune intention d'offenser ceux de sa propre race " $E l$ País, 6 septembre 1997). On retrouve ainsi cet évitement de la dimension raciale qui prend plusieurs formes : la dénonciation du racisme est interprétée comme une attaque contre les employées domestiques en général (“ Celles qui ont travaillé plusieurs années dans les foyers sont aussi indispensables qu'appréciées de tous les habitants de la maison "El País, 23 septembre 1997)) ou comme une critique contre les caricatures

(11) Nieves a pendant un temps hésité entre études de philosophie et de médecine... 
(“ on en terminerait avec les blagues et les caricatures E E País, 12 septembre 1997]). La logique de l'action de Pascual Charrupí est donc détournée, les critiques adressées à la tutelle ne portent pas sur son contenu. Ce décalage, stratégie utilisée pour éviter d'aborder directement la qualification raciale de l'autre, s'accompagne d'un discours de l'harmonie raciale qui euphémise les différences. " On mettrait un terme à ce sens de l'humour et de la fraternité de cette pluriethnie, dans laquelle nous ne sommes qu'une seule race, possédant toutes les couleurs "El País, 12 septembre 1997). Mieux même : la tutelle apparaît désormais comme une dévalorisation de la culture 'noire' voire même du qualificatif 'noir' en général.

“ J'espère que l'action de tutelle ne va pas aboutir parce que vous imagineriez faire une tutelle contre les sculpteurs de San Martín de Porres et contre la Negra de Chontaduro, ou contre le compositeur du 'Negrito del Batey' ou contre celui qui a découvert les trous noirs de l'univers. Messieurs, s'il vous plaît "El País, 4 septembre 1997).

Ces commentaires montrent à quel point la question raciale est évacuée des interprétations quotidiennes de la réalité. Ou plutôt, elle fait l'objet d'une naturalisation telle que les images deviennent des stéréotypes, que les préjugés deviennent des normes : il serait propre à cette culture, à cette identité 'noire' de danser mais aussi de servir. Dans son éditorial, Liliane de Levy, interprétant la discrimination raciale à la lumière de l'antisémitisme, sorte de modèle paradigmatique du racisme, en oublie finalement d'aborder la situation colombienne. Mieux : elle conclut son article en affirmant que les accusations portées contre Nieves, bien loin de contribuer à la lutte contre le racisme, se trompent de cible et de méthode.

“ La lutte contre le racisme est longue et compliquée, mais il vaut la peine de la mener sans baisser les bras... et sans tomber dans la paranoïa en affirmant qu'un personnage aussi exquis que l'est 'Nieves' peut donner une mauvaise image des noirs de Colombie "El País, 20 septembre 1997).

Et, finalement, c'est l'entreprise de revalorisation de Nieves elle-même - dont on va voir qu'elle constitue l'axe principal de la logique de récupération par le multiculturalisme - qui est privée de toute signification, disqualifiant toute prétention à l'ascension sociale et louant une sorte de sagesse populaire mythifiée et conservatrice : “ On peut donner un diplôme de professionnelle, et même un diplôme supérieur, à beaucoup d'employées domestiques, sans qu' elles soient passées par les salles de classe traditionnelles "El País, 23 septembre 1997). Bref, Nieves n'a pas à sortir de la place qui lui est assignée, menaçant ainsi d'une remise en cause de l'ordre social 'naturel'.

\section{3. "Nieves est le symbole de notre diversité culturelle " (entretien, 18 décembre 2002)}

En 2002, la mairie de Bogotá a lancé une campagne, dans le cadre du plan de développement de la ville, portant, notamment, sur le thème de la productivité : son personnage principal est Nieves, qui apparaît sur une série de quatre affiches présentant chacune quinze vignettes sur lesquelles Nieves se trouve dans une situation différente 
et délivre un message sur la productivité (12). Cette initiative est à replacer dans le cadre des campagnes civiques et pédagogiques initiées au cours du premier mandat d'Antanas Mockus à la mairie de Bogotá (entre 1995 et 1997) et reprises, de façon plus limitée, après sa deuxième élection (en octobre 2000). L'objectif, selon le responsable de la Consejeria Región y Competición de la mairie, chef d'orchestre de cette campagne publicitaire, est de faire sortir le thème de la productivité du seul domaine économique pour le transformer en préoccupation quotidienne. "Nieves parle de la productivité dans le langage de tout le monde, sans avoir besoin d'utiliser les équations de Samuelson " (Entretien, 18 décembre 2002) (13).

Le choix de Nieves répond à trois arguments principaux. Tout d'abord son “ appartenance à la race noire "Nieves incarnerait ainsi le nouveau visage multiculturel du pays dont Bogotá se veut le fer de lance. Ensuite le fait d'être une femme, mis en relation avec un fort courant féministe au sein de l'administration Mockus et avec une représentation de la femme comme plus responsable et pédagogue. Enfin, d'un point de vue plus technique, le fait que la caricature de Nieves s'accompagne de phrases courtes, facilement compréhensibles. Lorsqu'on l'interroge plus directement sur le 'passé' de Nieves et sur les dénonciations de certains militants, le fonctionnaire renverse immédiatement ces accusations en faveur de la campagne Nieves est avant tout l'histoire d'une jeune femme passée de la domesticité aux études et incarne ainsi une mobilité sociale et un désir d'éducation valorisés. D'ailleurs le fait que ce personnage, associé depuis sa naissance à Cali, devienne un symbole utilisé à Bogotá ne manque pas d'être significatif. Comme si l'ascension sociale et le déplacement géographique se combinaient dans un même mouvement de transformation du statut et d'inversion du stigmate. Comme si, d'une certaine façon, Nieves n'était plus tout à fait 'noire', comme le reconnaît le responsable du programme. Comme si seuls les traits stéréotypés positifs ou acceptables (innocence, acuité du jugement, soif d'apprendre) avaient été conservés.

En effet, ce qui est en jeu ici, c'est la reconnaissance d'une différence qui échapperait à la fois à la logique de l'infériorisation et à celle du blanchiment. Mais en troquant le tablier pour les livres de classe, Nieves ne cède-t-elle pas ainsi à l'illusion de la table rase, qui transformerait le 'noir' en 'afrocolombien', rejetant le racisme au rang d'archaïsme dépassé et l'évacuant alors des débats, politiques ou scientifiques ? Paradoxalement,Nieves est suffisamment 'noire' pour jouer le rôle de faire-valoir du multiculturalisme, mais pas trop non plus pour ne pas renvoyer à une image dépréciative. Aussi bien, si comme le suggère Michèle Wallace (1990), le multiculturalisme viserait à remplacer le 'primitivisme' (et la pensée postmoderniste les certitudes de la modernité), on peut se demander si, plus que de succession, il ne serait pas plus approprié de parler de coexistence contradictoire entre primitivisme et multiculturalisme, entre principes d'infériorisation et de valorisation, entre altérité exotisante et altérité bien-pensante.

(12) Ces vignettes ont également été visibles sur les factures d'électricité reçues par les habitants de Bogotá et dans les pages du quotidien national, largement centré sur Bogotá, El Tiempo.

(13) Les références qui suivent sont issues de cet entretien. 


\section{LES CONTRADICTIONS DU DISCOURS DE L'ETHNICITÉ}

Si les trois points de vue présentés précédemment rendent compte des modes ordinaires de relation à l' autre, les discours des militants 'noirs' et des anthropologues appartenant au courant des études afrocolombiennes livrent quant à eux des représentations qui, si elles sont certes plus limitées dans leur portée sociale, n'en témoignent pas moins de positions d'acteurs bénéficiant d'une certaine légitimité en matière de définition et d'usage des catégories de l'altérité.

\section{1. Acteurs ethniques}

Dans sa lettre adressée au tribunal supérieur de Cali, le 12 juillet 1997, Pascual Charrupí se présente comme professeur de l'Universidad del Valle et président de la Fundación Profesional para el Avance de la Raza Negra, FEDEPRAN. Il précise également que lui-même, ainsi que toutes les personnes ayant signé le document, appartiennent à la 'race noire'. L'objectif de la tutelle est donc d'interdire la publication de la caricature de Nieves dans le quotidien de Cali, El País. Elle est en effet accusée de diffuser un " message raciste " (14) laissant croire que " le seul destin et la seule aspiration de la femme noire sont d'être servante ", perpétuant ainsi l'idée que " les noirs ne sont faits que pour les tâches nécessitant une force physique brute ou pour le travail domestique, tâches qui furent à l'origine de l'importation de la main d'œuvre noire par les esclavagistes espagnols et créoles ". Ainsi, à travers la caricature, sont envoyés des " messages subliminaux porteurs de stéréotypes négatifs et d'infériorisation de la race noire ". La réitération de l'association entreNieves et l'occupation de servante est dénoncée comme une naturalisation, qui enferme toutes les femmes 'noires' dans un statut infériorisé. Dès lors, la transformation de Nieves, l'abandon du tablier pour les livres de philosophie, sont impuissants à transformer véritablement le stéréotype. Nieves, avec ou sans tablier, reste Nieves. “ Les femmes 'noires' étaient appelée\$ieves. C'était péjoratif. Elles pouvaient bien étudier, elles étaient toujours Nieves " (15). La qualification raciale agit ici comme un 'master status' (Hughes, 1994) qui prend le pas sur les autres traits identitaires et oriente, à partir d'un raisonnement en cascade, l'interprétation des actes et gestes observés. L'habit d'étudiante ne parvient pas, comme dans le discours du multiculturalisme, à effacer l'ancrage racial premier.

Par ailleurs, il est à la fois étonnant et révélateur de constater que l'action de Pascual Charrupí n'a rencontré aucun écho au sein du mouvement ethnique émergeant (16). Étonnant car les accusations de racisme faites à la caricature de Nieves auraient pu constituer la base d'une dénonciation plus générale dans un contexte d'affirmation du

(14) Selon le texte de la tutelle.

(15) Entretien avec Nestor Raúl, 30 août 2002, frère de Pascual Charrupí (ce dernier étant décédé dans un accident de voiture en 1999, ses deux frères, Nestor Raúl et Harold, ont repris le flambeau de la dénonciation du racisme en rassemblant notamment de nombreux documents laissés par Pascual - essais, lettres, texte de la tutelle et réponse des juges - en vue d'une publication).

(16) Précisons toutefois qu'il existe quelques (rares) références au personnage de Nieves dans certains écrits qui, comme ceux d'Alberto Angulo, se posent comme des militants alternatifs ou non institutionnels de la cause 'noire'. Il est d'ailleurs intéressant de remarquer que, à côté de la dénonciation de l'enfermement du 'noir' dans un statut inférieur (“ bien que l'esclavage ait été aboli 
multiculturalisme et du droit à la différence. Révélateur car le silence des leaders 'noirs' témoigne de certaines caractéristiques et difficultés du discours ethnique. C'est ainsi que l'un des arguments avancés pour justifier l'absence de mobilisation porte sur les ambitions strictement individuelles de Pascual Charrupí, pour lequel la tutelle aurait été un moyen d'obtenir une place au Sénat, la dimension raciale étant alors instrumentalisée et l'engagement collectif désamorcé. Face à ces accusations de détournement intéressé de la tutelle, la famille Charrupí renverse, quant à elle, les arguments : ce n'est pas tant l'action entreprise par Pascual qui aurait été maladroite que l'absence de soutien d'un mouvement ethnique impuissant qui aurait éclaté au grand jour. " Le mouvement afro est une illusion, entre les militants eux-mêmes il n'y pas d'union "Entretien, 30 août 2002). On retrouve ici cette tension entre projet d'ascension personnelle et mobilisation communautaire propre aux mouvements sociaux dans leur ensemble mais aussi aux manipulations autorisées par l'imprécision même du statut de 'noir'. Agustin Valencia, également originaire de Cali, à l'époque sénateur sur un poste réservé aux populations 'noires', rappelle ainsi qu'il n' a connu l'action de tutelle que par la presse et n'a jamais été contacté par Pascual Charrupí (Entretien, 22 juin 2002). Son analyse a posteriori est même extrêmement critique : la tutelle, en échouant, aurait finalement eu pour effet de renforcer la stigmatisation du 'noir' en légitimant son association à un statut inférieur et en déqualifiant ainsi toute dénonciation future du racisme. Juan de Dios Mosquera, président de Cimarrón, l'une des plus anciennes et plus actives associations 'noires', va encore plus loin : la tutelle était vouée à l'échec car elle se heurte à une " psychologie dominante d'intériorisation du racisme. Il faudrait que la population ait conscience que c'est du racisme. Mais elle le voit comme une jolie petite caricature folklorique. La société ne veut pas accepter que c'est une marque de racisme "Entretien, 25 juin 2002).

Au-delà des querelles de personnes et des enjeux de pouvoir, se pose la question de l'incapacité du mouvement 'noir' naissant à se rassembler autour du thème du racisme et à le faire émerger sur la scène publique. Seuls certains objectifs concrets, en particulier la titularisation des terres, ont été réalisés, dans un système normatif ou un “ cadre de l'expérience " pour reprendre le terme de Goffman qui demeurent en grande partie inchangés. De même que Nieves, avec ou sans tablier, reste Nieves, les 'communautés noires', désormais propriétaires de larges territoires dans le Pacifique, n'en demeurent pas moins associées à une représentation immuable du 'noir' qui s'appuie sur des stéréotypes sans cesse renouvelés.

\section{2. L'aveuglement de l'anthropologie}

Il est à première vue surprenant de constater que la revue América Negra, fondée par Nina de Friedemann, accorde, dans l'un de ses numéros, une large place à la caricature de Nieves : loin de dénoncer le racisme qui accompagne le personnage, la

il y a plus d'un siècle, [Consuelo Lago] fait comme si ses caractéristiques mentales s'étaient perpétuées au $21^{\text {ème }}$ siècle. Nieves est le symbole impérissable d'un esclavage perpétuel "(Angulo, 1999 :231), pointe une autre critique de Consuelo Lago qui chercherait à se réclamer du mythe — symétrique de la blancheur : “ elle donne l'impression qưieves aurait été créée par Isabelle la Catholique et non par une descendante directe des indiens abusés "(Angulo, 1999 :230). Fait symptomatique néanmoins : l'auteur ne fait aucune référence à la tutelle de Pascual Charrupí. 
revue en fait au contraire un des éléments de cette 'identité africaine' à la recherche de laquelle seraient vouées les études afrocolombiennes. Vêtue à la mode africaine, Nieves incarne, de façon positive, les 'empreintes d'africanité' (huellas de africania), objet de la nouvelle anthropologie afrocolombienne, permettant d'oublier le préjugé racial qui oriente la caricature. Dans l'éditorial du numéro 12América Negra, 1996) de la revue, entièrement consacré à Nieves, Nina de Friedemann voit en la présence de la caricature dans les pages d'América Negra une double victoire pour les populations 'noires' : non seulement elle favorise la 'visibilisation' de la présence africaine en Colombie, mais elle témoigne également du nouveau visage multiculturel du pays.

“América Negra est fière de présenter Nieves et Hétor, ces personnages qui ont, depuis trente ans, rendu visible la présence historique de la racine africaine dans la quotidienneté nationale. Ce numéro célèbre le nouveau visage de la diversité sociale et culturelle du pays, à travers l'expression artistique de Consuelo Lago " (América Negra, 1996 : 6).

Concentrés sur le travail de sauvegarde et de valorisation d'une culture qui serait ancestrale et authentique, les chercheurs d'América Negra en oublient ainsi que Nieves ne joue pas seulement, le temps de son passage dans la revue, le rôle d'une reine africaine (17) : chaque jour, elle endosse les habits d'employée de maison. Sans doute l'insistance sur le voyage qui mène de l'Amérique à l'Afrique “ afin de visiter les moindres recoins du Congo, de l'Angola, du Sénégal, de la Côte de l'Or, de la Côté d'Ivoire et ces autres sites de la fabuleuse Afrique "América Negra, 1996 : 6) répond-elle à des critères scientifiques pertinents et à une revendication politique légitime ; il n'en demeure pas moins que, tout à son exotisme mystérieux, elle occulte la réalité quotidienne, faite de discriminations et de stéréotypes bien plus que de " mémoires anciennes de croyances, de proverbes et de saveurs "América Negra, 1996 : 6). Vêtue à l'africaineNieves n'est plus 'noire' mais 'afrocolombienne' ou 'afrodescendante'. Et il n'est plus question de racisme, mais de préservation de l'héritage africain et de valorisation de l'identité ethnique. On comprend alors pourquoi la représentation de Nieves ne dérange pas les éditorialistes d'América Negra : car il n'est pas question de race - et tout ce qui l'accompagne : discrimination, stéréotype, préjugé — mais d'ethnicité, définie en termes culturels et généalogiques.

Aussi bien, la fameuse 'invisibilité' qui caractériserait les populations 'noires' avant 1991, loin de signifier absence de catégorisations raciales et de discours sur l'autre, correspond avant tout à l' absence des critères de définition tels qu'ils apparaissent avec l'introduction du multiculturalisme. L'invisibilité n'est pas tant ici dans l'objet que dans le regard qui ne peut pas ou ne veut pas voir. Et l'ethnicité, autant qu'une nouvelle catégorie identitaire, devient alors un objet légitimant une analyse scientifique spécifique, donnant naissance au courant des 'études afrocolombiennes'. La quête des traces

(17) Les caricatures d'América Negra la représentent en Reine Ginga de Matamba et Angola, en joueuse de tambour, en jeune fille de Cabinda en Angola, etc. Lorsqu'elle apparaît avec son tablier, Nieves se cache le visage sous une serviette et affirme : "j' ai une angoisse anthropologique 'Anérica Negra, 1996 : 132). Tout se passe comme si l'absence de signes extérieurs africains signifiait automatiquement un déchirement identitaire, s'exprimant lui-même, matériellement ici, par la recherche d'invisibilité. 
d'africanité, si elle s'inscrit dans une orientation théorique que certains chercheurs, en Colombie, qualifient d' 'essentialiste' par opposition à une approche considérée comme 'post-moderniste' (ou constructiviste) a deux conséquences fondamentales : d'une part, en même temps qu'elle l'étudie, elle produit aussi son propre objet de recherche (l' 'ethnicité afrocolombienne') ; d'autre part, débordant le champ scientifique, elle influe le discours des acteurs sociaux, fonctionnaires des administrations et militants 'noirs' principalement. Si ce processus de diffusion de la science vers la pratique est loin d'être mécanique et linéaire, il n'en demeure pas moins que la politique multiculturelle mise en place par le gouvernement colombien fut inspirée par une vision statique et discontinue de la culture, en parfaite adéquation avec le discours scientifique, faisant de l'association 'identité-territoire' le préalable à toute revendication politique.

En réduisant le champ de la recherche à l'étude des traces d'africanité en Colombie, d'autres thèmes d'analyse, jugés non pertinents, sont ainsi évacués de l'agenda scientifique, mais aussi du discours des acteurs politiques et sociaux. On ne sera alors pas étonné de constater à quel point le 'renforcement de l'identité afrocolombienne' est devenu un préalable à toute action politique. De fait, lorsque certains anthropologues, relayés par des dirigeants associatifs, appellent à éliminer du langage quotidien et scientifique le terme 'noir' pour le remplacer par celui d' 'afrocolombien' (18) et, plus récemment, d' 'afrodescendant', ne supposent-ils pas que l'ethnicité pourrait se substituer aux catégories locales bien plus que s'ajouter à elles ? L'inversion de la signification associée au 'noir' à travers sa transformation en 'afrocolombien', le passage de la race, identifiée par la couleur, à l'ethnicité, définie par l'appartenance culturelle et le projet politique, ne conduisent-ils pas à minorer la permanence des stéréotypes liés aux apparences et à imposer une catégorisation sans signification pour la majorité de la population (19) ? Aussi bien, de façon paradoxale, ne pourrait-on pas affirmer que le processus d'ethnicisation qui accompagne l'affirmation du multiculturalisme agit comme une forme de 'naturalisation' des différences culturelles ? Le multiculturalisme, opposé au racisme, finit par créer de nouvelles formes d'exclusion : sa logique n'est plus d'exclure pour conserver l'identité d'un groupe dominant, mais d'exclure pour conserver les particularités culturelles d'une minorité (Taguieff, 1995). Autre conséquence : le racisme, quotidien et paternaliste, est oublié, relégué à un vestige de l'époque coloniale.

(18) “ Nous ne pouvons plus continuer à utiliser ces termes racistes aujourd'hui. Nous ne pouvons plus continuer à parler comme les esclavagistes et à reproduire ce langage dénigrant envers la personne africaine esclavisée. La nouvelle vision de notre Africanité et de notre Afrocolombianité, la nouvelle vision critique de l'histoire et des sciences sociales, l'engagement dans un discours éthique et politique des droits de l' homme, nous appellent à assumer la tâche de critiquer et contextualiser le langage dont nous avons hérité et de valoriser notre Afrocolombianité, en rendant aux personnes afros leur dignité humaine et leur identité culturelle africaine, ignorées et outragées. Nous devons tous apprendre que les personnes noires sont des personnes afros, afrocolombiennes " (Mosquera, 2000 : 6-7).

(19) Ces interrogations sont reproduites à l'intérieur même de DANE, institut de statistiques colombien, qui, au sujet de la mise en place d'un recensement ethnique, oscille entre définition culturaliste des identités basée sur la référence à l'ethnicité et définition phénotypique faisant référence à la race. 


\section{CONCLUSIONS}

Nieves nous informe ainsi sur les mécanismes de la discrimination raciale produite par le discours de l'élite, mais aussi sur sa diffusion sociale, à tel point que les stéréotypes infériorisants utilisés pour qualifier le 'noir' deviennent ordinaires, normaux, 'naturels', alors que l'inversion des identifications opérée par le multiculturalisme s'ajoute aux anciens cadres normatifs sans véritablement les remettre en cause. Nieves révèle également les ambiguïtés du mouvement politique et social 'noir' naissant qui n'a pas pu ou pas voulu profiter de l'action de tutelle émise en 1997 pour dénoncer symboliquement la discrimination à l'œuvre dans le pays. Nieves, enfin, montre à quel point l'anthropologie colombienne, à la recherche de l'Afrique en Amérique Latine, est restée hermétique à la question du racisme et du rôle social des qualifications raciales, l'excluant du champ scientifique légitime et, au-delà, de l'agenda politique du multiculturalisme.

En guise de conclusion je reviendrai rapidement sur les trois points théoriques présentés en première partie et sur l'éclairage que leur apporte la caricature de Nieves. Tout d'abord, le racisme (qui se redouble ici de sexisme) est de l'ordre du non dit omniprésent, la race, loin d'être un terme banni du langage quotidien, est une catégorie sociale de classement d'autant plus présente qu'elle est implicite, d' autant plus effective qu'elle est diffuse. En ce sens, on peut se demander si Nieves serait plus impatiente de connaître “ son ancêtre africain 'A(nérica Negra, 1996 : 5) comme le suggère Nina de Friedemann que de bénéficier des mêmes opportunités que ses contemporains colombiens Nieves avec une identité n'est-elle plus victime du racisme ? En d'autres termes, l'insistance actuelle des différents acteurs (militants 'noirs', presse, représentants des administrations), légitimée par le discours scientifique, sur les notions d'identité, ethnicité, multiculturalisme, ne tend-elle pas à évacuer la problématique de la discrimination raciale et le rôle social des apparences physiques, dans une logique de reproduction et de perpétuation de l' 'invisibilité' du 'noir' ?

Par ailleurs, le stéréotype agit ici comme un schème cognitif qui offre des catégories préconstruites Nieves, peut-elle incarner, commel'affirment les responsables de la campagne lancée par la mairie de Bogotá, le nouveau visage multiculturel du pays ? Ou bien est-elle irrémédiablement perçue à travers le filtre de l'image associée au 'noir' ? Réussit-elle à endosser les habits de l'étudiante ? Ou reste-t-elle la domestique au service de l'élite 'blanche' ? Plus précisément, ne serait-elle pas à la fois étudiante et servante, 'blanche' et 'noire' ? Les stéréotypes incorporés, fonctionnant comme autant de préconstruits de la pensée, coexistent, paradoxalement, avec de nouvelles représentations qui leur sont bien souvent contradictoires. On comprend alors comment Vanessa Mendoza, première reine de beauté nationale 'noire', peut devenir à la fois le symbole d'une nation multiculturelle qui accepterait l'égalité dans la différence et l'incarnation de cette 'autre Colombie', sous-développée, en retard, sauvage. Ou comment la champeta, musique 'noire' de Cartagena, est présentée comme la nouvelle musique caribéenne de Colombie, sorte de synthèse de la diversité culturelle du pays et de la région, mais aussi comme une musique dangereuse, associée au sexe, à la violence, à la déviance. Exclusion et inclusion, identité et altérité, primitivisme et multiculturalisme se superposent ainsi dans un processus qui accentue le brouillage des statuts. 
Enfin, la réflexion sur les apparences nous montre que 'ce qui est donné à voir' est de l'ordre de l'image-connaissance, de l'évidence descriptive et normative. Mais également que 'ce qui est vu' dépend du regard de celui qui observe, incapable, aujourd'hui encore, tant pour les militants associatifs que pour les anthropologues afrocolombianistes, de voir la couleur noire pour ce qu'elle est, avec ses connotations sociales et culturelles dont la charge n'est pas désamorcée. Tout se passe comme si la différence était à la fois niée (le 'noir', invisible, ne fait pas 'saillance', il n'est pas un élément pertinent de l'appréhension de l'autre) et si grande qu'elle en devient indescriptible, prérequis, non questionné et non questionnable, des mécanismes de classement.

\section{Remerciements}

Je souhaite remercier tout particulièrement Fernando Urrea, professeur de l'Universidad del Valle à Cali, qui est à l'origine de cette recherche, ainsi que toutes les personnes ayant eu la gentillesse de répondre à mes questions.

\section{Références citées}

AMÉRICA NEGRA, 1996 - º12, décembre.

ANGULO, A., 1999 - Moros en la Costa. Vivencia afrocolombiana en la cultura colectiva, 237p.; Bogotá : Docentes Editores.

APPADURAI, A., 2001 - Après le colonialisme. Les conséquences culturelles de la globalisation, 322p. ; Paris : Payot.

BALIBAR,E.\&WALLERSTEIN,I., 1990 -Race, nation, classe.Lesidentitiésambiguës, 307p. ; Paris : Édition La Découverte.

BOETSCH, G. \& VILLAIN-GANDOSSI, C., 2001 - Introduction. Hermès. Cognition. Communication. Politique, $30: 17-23$.

BOURDIEU, P., 1998 - La Domination masculine, 142p. ; Paris : Seuil.

BRUBAKER, R., 2001 - Au-delà de l' 'Identité'. Actes de la Recherche en Sciences Sociales, 139 : 66-85.

CHEBEL D'APPOLLONIA, A., 1998 - Les racismes quotidiens, 109p. ; Paris : Presses de Science-Po - Bibliothèque du Citoyen.

ESSED, P., 1991 - Understanding everyday racism: an interdisciplinary theory, 336p. ; Newbury Park-London-New Delhi : Sage.

FRIEDEMANN, N. de, 1993 - La Antropología Colombiana y la Imagen del Negro. América Negra, 6 : 161-172.

GLISSANT, E., 1997 - Traité du Tout-Monde, Poétique IV, 262p. ; Paris : Gallimard.

GOFFMAN, E., 1973 - La mise en scène de la vie quotidienne. La présentation de soi (tome 1), 255p. ; Paris : Les Éditions de Minuit.

GUILLAUMIN, C., 1992 - Sexe, race et pratique du pouvoir. L'idée de nature, 239p. ; Paris : Côté-femmes éditions.

HUGHES, E. Ch., 1994 - Dilemmas and Contradictions of Status. The American Journal of Sociology, L, 1 : 353-359.

LAGO, C., 2001 - Nieves impertinente y coqueta, 142p. ; Bogotá : Villegas Editores.

MERLEAU-PONTY, M., 1964 - Le visible et l'invisible, 359p. ; Paris : Gallimard. 
MOSQUERA, J. de D., 2000 - Racismo y discriminación racial en Colombia ; Bogotá : Docentes Editores.

SAID, E., 1978 - Orientalism, 416p. ; New York : Pantheon Book.

TAGUIEFF, P.A., 1995 - Les Fins de l'anti-racisme, 708p. ; Paris : Michalon.

VILLAIN-GANDOSSI, C., 2001 - La genèse des stéréotypes dans les jeux de l'identité/ altérité Nord-Sud. Hermes. Cognition. Communication. Politique, 30 : 27-40.

WACQUANT, L. J. D., 1997 - Three Pernicious Premises in the Study of the American Ghetto. International Journal of Urban and Regional Research, 21(2) : 341-353.

WALLACE, M., 1990 - Modernism, postmodernism and the problem of the visual in AfroAmerican culture. In :Out there: marginalization and contemporary cultures(Ferguson, R.éd.):39-50 ; New York-Cambridge-London : The Museum of Contemporary Art- The MIT Press. 